ELUA

ISSN 2171-6692

Núm. 36, 2021, págs. 67-87

https://doi.org/10.14198/ELUA.2021.36.04

\title{
Propuesta de formalización de las locuciones nominales para un diccionario electrónico español
}

\author{
Proposal for the formalization of nominal idioms for a spanish \\ electronic dictionary
}

\author{
María Auxiliadora Barrios Rodríguez \\ Universidad Complutense de Madrid, España \\ auxibarrios@filol.ucm.es \\ https://orcid.org/0000-0001-6830-4797
}

\begin{abstract}
Resumen
Las locuciones nominales del español constituyen un rico recurso para la evaluación de personas, entidades y acontecimientos, pero no siempre hay acuerdo en cuanto a su categorización semántica y sintáctica. Su clasificación debería ser sistemática y coherente, y así se facilitaría su inclusión en cualquier recurso lingüístico. Sin embargo, las diversas fuentes ofrecen datos que pueden resultar contradictorios para cualquier desambiguador morfosintáctico o semántico. Así, por ejemplo, los diccionarios de uso del español recogen estas locuciones agrupadas por su forma, de modo que en la entrada canela se puede encontrar canela en rama y canela en polvo, pero la polisemia de canela en rama (tipo de canela, o alguien o
\end{abstract}

\begin{abstract}
Spanish nominal idioms are adequately rich to constitute a vast range of expressions of evaluation and judgment both about people and situations, however this predicative use of idioms is not so explicit in the dictionaries. In fact, Spanish dictionaries collect this kind of idioms grouped by their form: for instance the entry of the dictionary for canela (cinnamon) includes canela en rama (stick cinnamon) and canela en polvo (cinnamon powder). This methodology of collection of idioms is correct but not useful for the semantic analysis of non-literal senses: actually canela en rama has three senses in Spanish: 1. stick cinnamon; 2. delightful person; 3. outstanding object. Electronic dic-
\end{abstract}

Esta investigación se ha realizado en el curso del proyecto "Diretes: Diccionario Reticular Español. Diccionario analógico y relacional con acceso en red desde el sentido y desde la forma" (FFI-2017-83293), financiado por el Ministerio de Ciencia, Innovación y Universidades, y por fondos europeos (FEDER). Agradezco a los revisores todas sus sugerencias y comentarios. Cualquier aspecto con el que pueda no estar de acuerdo el lector es responsabilidad única de la autora.

Para citar este artículo: Barrios Rodríguez, M. A. (2021). Propuesta de formalización de las locuciones nominales para un diccionario electrónico español. ELUA, (36), 67-87. https://doi.org/10.14198/ ELUA.2021.36.04

Recibido: 06/08/2021, Aceptado: 08/10/2021

(C) 2021 María Auxiliadora Barrios Rodríguez

Este trabajo está sujeto a una licencia de Reconocimiento 4.0 Internacional de

Creative Commons (CC BY 4.0) 
algo excelente) solo se refleja en la definición. Los diccionarios electrónicos precisan de información semántica y sintáctica, por lo que se debe hacer explícito que el primer sentido de canela en rama funciona como un sustantivo y el segundo como un adjetivo. Aquí nos servimos de una tipología y de un modo de formalizar las locuciones nominales del español creada en el marco de la Teoría Sentido-Texto y basada en el análisis semántico de un corpus de setecientas setenta y seis construcciones nominales. Las locuciones se clasifican de modo formal mediante unas funciones léxicas creadas ad hoc. Esta propuesta se ha implementado en el diccionario electrónico del español Diretes. Gracias a la formalización mencionada, se puede navegar en el diccionario en sentido inverso al habitual, de modo que desde bueno se puede llegar a excelente, y desde excelente a canela en rama. Se presentan algunos resultados y se concluye que, a pesar de lo reducido del corpus analizado, la implementación en el diccionario permite validar la tipología de las locuciones nominales con la que se ha trabajado y la formalización de funciones léxicas propuesta.

PALABRAS CLAVE: Locuciones nominales; palabras compuestas; uso predicativo; diccionario electrónico; Teoría Sentido-Texto. tionaries demand a formal and correct way to show nominal idioms' semantic features and their syntactic structure. However, sources on which e-dictionaries are based offer data that can be contradictory for any disambiguator. In order to solve these problems, this paper proposes a new formalization of nominal idioms within the Meaning-Text Theory, according to their semantic and syntactic behavior. It is based on a corpus of seven hundred and seventy-six nominal idioms, which was created ad hoc for Diretes, a Spanish electronic dictionary. This proposal allows the readers of the dictionary to arrive at non-literal senses: for instance, the reader can arrive at canela en rama (stick cinnamon) not only from the word canela (cinnamon), as in other dictionaries, but from the adjectives extraordinario (delightful) and excepcional (otustanding). The paper presents some results and concludes that, despite the small size of the corpus, this work is a first step for the validation of the proposed typology of nominal idioms.

KEY WORDS: Nominal idiom; compound noun; predicative use; e-dictionary; MeaningText Theory.

\section{INTRODUCCIÓN}

Las locuciones nominales y su tratamiento en los diccionarios despiertan interés no solo en el ámbito fraseológico sino también en áreas como la de Procesamiento de Lenguaje Natural (PLN). Aunque en los diccionarios de uso general no se suele prestar especial atención a estas construcciones, la investigación en ámbitos relacionados con la inteligencia artificial demuestra que su tratamiento lexicográfico es particularmente importante: no solo tiene repercusión cómo se refleja la forma de las locuciones nominales en los diccionarios sino también cómo se describe su significado. Como botón de muestra del interés que suscita, mencionaremos el hecho de que en la investigación de datos enlazados se recurra habitualmente a ontologías, diccionarios y enciclopedias (Bosque-Gil et alii 2016, 2018; Ionov et alii 2020). En su consulta a las citadas fuentes los investigadores tropiezan con dos escollos. El primero es que los diccionarios a los que acceden no están disponibles en lenguajes apropiados al modelo de datos para metadatos del Marco de Descripción de Recursos (en inglés Resource Description Framework, RDF) (Bosque-Gil et alii 2016). El segundo problema es que, hasta el momento, dichos diccionarios fueron creados para seres humanos (Barrios Rodríguez 2020) y, por lo tanto, en ellos las locuciones se describieron de modo no sistemático, por lo que recuperar la información semántica de modo automático resulta una tarea muy difícil. 
En los últimos cinco años se ha ido consolidando Ontolex-Lemon como un modelo estándar para la representación léxica (McCrae et alii 2017, Bosque-Gil et alii 2019). Es relevante para nuestra propuesta que este modelo sea compatible con las Funciones Léxicas (FL) de la Teoría Sentido-Texto (TST) (Mel'čuk, 1996, 2014; Mel’čuk y Wanner, 2001), pues dichas funciones se revelan como una de las herramientas que, desde el ámbito de la lingüística, se ha ofrecido con más potencial para aplicaciones de PLN (Fontenelle 2000; Wanner y Lareau 2009; Fonseca et alii 2016a, 2016b, 2017).

A este respecto se ha señalado que, precisamente en PLN, una de las mayores dificultades reside en que las relaciones semánticas no hayan sido sistematizadas (Mikolov et alii 2013). Roukos (2014) insiste en esta idea y señala que el problema no es la gramática, sino el modelo: "lo que necesitamos es la formalización de significados útiles"1. En estos últimos años, gracias a la Representación de Significado Abstracto, se ha dado un vuelco en el análisis semántico (Mihindukulasooriya et alii 2020). El aprendizaje profundo y, entre otros, el programa BERT, están permitiendo un enorme avance, aunque se reconoce que desde el punto de vista lingüístico aún precisa de mejoras (Pilehvar et alii 2019). En aplicaciones bilingües se han creado algoritmos que reconocen la granularidad semántica entre parejas de palabras equivalentes en dos lenguas con un $80 \%$ de exactitud (Kouvara et alii 2020). Sin embargo, a pesar de los avances, se reconoce que sigue siendo un problema la distinción de los pequeños matices semánticos que afloran en el plano léxico y que afectan al plano oracional. Hasta el momento esto solo se puede solucionar con anotación manual (Kouvara et alii 2020).

A este respecto, el problema fundamental estriba en que dicha anotación no siempre puede hacerse de modo sistemático. Precisamente uno de los mayores obstáculos se relaciona con las locuciones nominales, cuya categoría gramatical (su nombre indica que funciona como un nombre) no siempre es paralela a su verdadero funcionamiento (muchas de ellas funcionan como adjetivos) (Barrios 2022). ¿Cómo anotarlas? Y una vez anotadas, ¿cómo comprender sus matices semánticos para representar de un modo objetivo, formal y coherente, qué significa la niña de sus ojos, mirlo blanco o cabeza hueca?

Toda la bibliografía precedente reconoce que, entre las construcciones nominales del español, se encuentran las colocaciones nominales, los compuestos sintagmáticos y las locuciones nominales. Con respecto a las colocaciones y al fenómeno combinatorio, la abundante bibliografía no es unánime ni siquiera en el propio concepto de colocación (véanse, entre otros, Bosque 1989, 1999, 2004; Mel'čuk 1996, 2014; Koike 2001; Polguère 2007; Torner y Bernal 2017; Buendía-Castro 2020). Acerca de los compuestos sintagmáticos también hay discrepancias en no pocos casos (véanse, entre otros, Zuluaga 1980; Bustos Gisbert 1986; Bosque 1989, 1999, 2004; Buenafuentes de la Mata 2007, 2010; García Page 2008, 2011, 2012; RAE, 2009; Koike, 2009; Alonso Ramos 2009; Cabezas García 2020).

Pero donde más dificultades afloran es en el estudio de las locuciones nominales. Los trabajos teóricos y lexicográficos muestran su riqueza y prueban sobradamente la dificultad de su clasificación (Casares 1969; Corpas Pastor 1996; Seco et alii 2004; Mel'čuk 2006; García Page 2008; Penadés 2008; Orduña López 2019). Consideramos que los desarrollos potencialmente útiles en PLN permiten validar propuestas lingüísticas teóricas (Barrios Rodríguez 2010), por eso vemos la sistematización de las locuciones nominales como un candidato óptimo para un trabajo de implementación como el que pretendemos aquí.

1 La cita que mencionamos la tomamos de la conferencia oral, disponible en https://www.youtube.com/ watch?v=a4ooQT2osOQ. 
En efecto, eso es lo que hacemos: partimos de una tipología de locuciones nominales (Barrios Rodríguez 2022), proponemos la creación de FL no estándar para la formalización de las mismas y para su inclusión en un diccionario de uso general, y mostramos los resultados de dicha implementación en el diccionario Diretes. Nuestro objetivo es responder a estas dos preguntas: ¿Es válida dicha tipología? ¿Es adecuada la propuesta de formalización de las locuciones nominales con las funciones léxicas no estándar que hemos creado ad hoc y presentamos en este trabajo?

Este artículo se organiza del siguiente modo: en el apartado 2 se describe brevemente el diccionario electrónico en el que estamos trabajando, Diretes; en el apartado 3 se sintetiza cómo se formalizan las relaciones léxicas en la Teoría Sentido-Texto y se compendian las características de su herramienta estrella, las Funciones Léxicas; en el apartado 4 se resume la metodología que hemos seguido y presentamos las Funciones Léxicas no estándar usadas en nuestro trabajo; en el 5 se explica cómo hemos formalizado las que llamamos locuciones nominales predicativas, y en el 6 , las locuciones nominales designativas; por último, en el apartado 7 se presentan los resultados y en el 8 las conclusiones.

\section{DIRETES VS. OTROS DICCIONARIOS}

Diretes es un diccionario electrónico que estamos desarrollando en la actualidad con contenidos formalizados en el lenguaje de la TST (Barrios Rodríguez 2019a, 2020; Barrios Rodríguez et alii 2019)². En este modelo se codifican las relaciones entre piezas léxicas (como ramo de rosas) mediante un lenguaje formal (en este caso, Mult(rosa) = ramo (de)), tal como veremos en (2) en el apartado 3.

En nuestro diccionario trabajamos con el modelo de la TST porque, a nuestro juicio, este modelo tiene un enorme potencial en todo lo relativo a relaciones léxicas y semánticas, incluidas las locuciones. Esto se pone de manifiesto particularmente cuando se comparan los diccionarios de la lexicografía tradicional con los diccionarios desarrollados en la TST. Entre estos últimos hemos de mencionar el diccionario combinatorio del francés Dicouèbe ${ }^{3}$, el diccionario electrónico Réseau Lexical du Français ${ }^{4}$ (RLF) (Polguère 2015), el diccionario del español $D I C E^{5}$, y el traductor automático ETAP-46 (Apresjan et alii 2003).

El potencial de la TST con respecto a la fraseología se aprecia fácilmente al consultar cualquiera de estos diccionarios porque, mientras que en los diccionarios tradicionales de uso no encontramos más que algunas colocaciones aisladas y, generalmente, no reconocidas como tal, en la TST se recogen y formalizan todas las colocaciones. Así, por ejemplo, ramo de rosas y gota de agua no están en el Diccionario de Lengua Española (DLE $)$, pues su transparencia en el significado fuerza que se vea como innecesario. En los diccionarios de la TST, sin embargo, se encuentran estas y otras muchas colocaciones, pues se considera que las colocaciones expresan un sentido general, compartido por otras muchas lenguas,

2 Por el momento se puede consultar una prueba piloto del diccionario en http://diretes.es/ (26/10/2021).

3 Disponible en la página web: http://olst.ling.umontreal.ca/dicouebe/index.php

4 Disponible en la página web: https://lexical-systems.atilf.fr/spiderlex/

5 Disponible en: http://www.dicesp.com/paginas. Este diccionario por el momento contiene solo el léxico de las emociones del español. Las diferencias con Diretes se explican en Barrios Rodríguez (2020).

6 Disponible en: http://cl.iitp.ru/

7 Disponible en: https://www.rae.es/. 
pero no siempre expresado del mismo modo. Así, por ejemplo, la colocación del español ramo de rosas es ambigua para un sinoparlante, ya que en chino se sirven de un clasificador, y distinguen entre el objeto 'ramo de rosas' y el sentido 'cantidad de rosas que hay en un ramo' (Liu 2018).

En relación con las locuciones, en los diccionarios de uso (particularmente en los impresos), salvo en el Diccionario de uso del español (DUE, Moliner 2016), solo se reflejan dichas construcciones en el lema de algunas de las palabras: de este modo, por ejemplo, la locución mirlo blanco no origina una entrada de diccionario, sino que se presenta en la entrada mirlo ${ }^{8}$. En los diccionarios de la TST, sin embargo, las locuciones, además de vincularse a las entradas de alguna de las palabras que la constituyen, tienen su propia entrada, en la que se reflejan todas las relaciones léxico-semánticas asociadas a cada locución. En el caso de la entrada bon, del $R L F$, por ilustrar lo que decimos, hay una decena de locuciones en las que aparece esta palabra, como bonne humeur, bon sens, de bonne famille, bonne mère, etc. Si se accede a las entradas de estas locuciones, se encuentran otras relaciones semánticas para cada una de ellas (por ejemplo, se refleja que bonne mère es sinónimo de Mère de Dieu).

Es cierto que en los diccionarios de uso habitual que tienen formato electrónico, hay información adicional gracias a los hipervínculos, pues estos crean enlaces entre las palabras de la definición y otras entradas del diccionario. Como permiten llegar a otros lemas, resultan especialmente útiles en el caso de las locuciones: así ocurre con mirlo del DLE, que contiene la locución nominal mirlo blanco, desde la que se puede llegar a rareza y a extraordinario. En (1) reflejamos dicha entrada:

(1) Mirlo

- Mirlo blanco

1. m. Persona de rareza extraordinaria9.

Esta facilidad del $D L E$ para llegar desde mirlo al lema extraordinario mediante hipervínculos resulta, sin embargo, bastante limitada. Como señala Bosque, meter la pata significa 'equivocarse', pero desde esta locución no se puede llegar a la entrada del verbo equivocarse porque en el $D L E$ su definición es "hacer o decir algo inoportuno o equivocado", de modo que solo se podrá encontrar el vínculo entre meter la pata y el adjetivo equivocado (2017). Y esto es así porque en los diccionarios de uso se trabaja con un enfoque semasiológico (solo se puede llegar a la definición desde el lema), y no con un enfoque onomasiológico (que permitiría llegar a las locuciones desde su significado). Si se ofreciera este último enfoque, la persona que consultara el diccionario podría buscar la entrada equivocarse y encontrar todos sus cuasi-sinónimos, entre ellos meter la pata.

Bien pensado, en el caso particular de las locuciones, resultaría especialmente relevante dar un salto del enfoque semasiológico al onomasiológico, pues ese contenido semántico es relevante para llegar a otras formas de expresar un mismo sentido. Pero además, con una perspectiva más amplia, sería muy útil que el diccionario incluyera otras relaciones léxicas:

8 Un caso aparte es el de los diccionarios de locuciones, en los que precisamente los lemas del diccionario son locuciones.

9 Mirlo blanco, aunque no lo refleja esta entrada del $D L E$, tiene connotaciones muy positivas y se usa para hablar de alguien extraordinario. 
así, en el caso de mirlo blanco, se trataría de permitir llegar a esta locución no solo desde el lema mirlo (como ocurre en los diccionarios tradicionales impresos) o desde la entrada excepcional (como ocurre en los de la TST y en el DUE), sino también desde la relación de mirlo blanco con la locución canela en rama, y con el adjetivo excelente, todos ellos vinculados por la cuasi-sinonimia.

En nuestro diccionario Diretes tratamos de que todas las locuciones se reflejen de manera que se pueda trabajar con este enfoque: desde la forma se puede acceder al contenido, y desde el contenido se puede acceder a la forma (Barrios Rodríguez 2020) ${ }^{10}$. Intentaremos mostrar en este artículo cómo lo hacemos al tiempo que abordamos cómo hemos clasificado las locuciones nominales para permitir dicho acceso múltiple. Adelantamos ya que nuestra propuesta no se basa en un recurso tecnológico (como ocurre con los hipervínculos) sino en la formalización del significado de la locución, como veremos a continuación. Pretendemos formalizar la semántica de las locuciones nominales, de forma que su etiquetado sea coherente desde el punto de vista sintáctico y semántico.

\section{FUNCIONES LÉXICAS Y TEORÍA SENTIDO-TEXTO}

A nuestro juicio, la Teoría Sentido-Texto resulta óptima para el propósito de nuestro trabajo, pues precisamente en la TST resulta de vital importancia el sentido onomasiológico: lo prioritario en este enfoque es el sentido que los hablantes tienen en su cabeza, y esto orienta todo el modelo (Mel'čuk 1996).

El recurso más conocido en la TST es el de la Función Léxica (FL), una suerte de fórmula que pone en relación un sentido abstracto, general y bastante productivo de una lengua con dos o más palabras de esa lengua. Así, como adelantábamos en el apartado 2, una colocación española como ramo de flores, puede expresarse en el lenguaje formal de la TST tal como se muestra en (2.a), donde Mult es una FL que significa 'conjunto de'. El caso de gota de agua se formalizaría tal como se muestra en (2.b), donde se aprecia que recurrimos a la FL Sing, que significa 'parte mínima de':

(2) a. Mult(flores) $=$ ramo (de)

b. $\operatorname{Sing}($ agua $)=$ gota $($ de $)$

Estas dos colocaciones nominales son solo dos ejemplos de un riquísimo repertorio de otras colocaciones cubiertas por Mult, como rebaño de ovejas, tripulación del avión o racimo de uvas, y Sing, como grano de arroz, copo de nieve o mota de polvo (Barrios Rodríguez 2010, 2015).

Las FL están tipificadas y se ha reconocido un repertorio de unas sesenta y cinco FL llamadas estándar, entre las que se encuentran Mult (2a) y Sing (2b). Son muy productivas en muchas lenguas y además se pueden combinar entre sí. Por ejemplo, dada la FL Magn (que significa 'muy/mucho') y la FL Bon (que significa 'bueno'), podemos combinarlas para obtener la FL MagnBon (que significa 'muy bueno'). Este es el sentido general que cubriría la colocación que se muestra en (3):

10 Diretes se ha diseñado como otros diccionarios creados en la TST, como diccionario electrónico y de acceso múltiple (Polguère, 2015). 
(3) MagnBon(cabeza) = lúcida

Además de las FL estándar, en la TST se trabaja con FL no estándar, funciones que han demostrado ser productivas solo en alguna lengua. Es el caso de Essayer, que significa 'intentar', propuesta por Polguère (2007) para el francés. En Diretes hemos comprobado que también es productiva en español, por lo que la usamos tal como se muestra en (4), donde se recurre a la FL CausContFunc ${ }_{1}$, que se puede parafrasear por 'causar que eso siga existiendo para el primer actante', e IntentarLiquFunc ${ }_{0}$, que se parafrasea por 'intentar que eso deje de existir' ${ }^{11}$ :

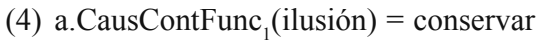

b.IntentarLiquFunc ${ }_{0}($ hipocresía $)=$ denunciar

Hasta ahora, que sepamos, en la TST no se ha propuesto ninguna formalización con FL no estándar, similar a la de (4.b), para las locuciones nominales. Lo cierto es que estas locuciones, por su doble función nominal y adjetival, reclaman un análisis más detallado y fino que otro tipo de locuciones, y nos parece acertado, a priori, intentar crear alguna FL no estándar que permita reflejar mejor sus características semánticas y sintácticas.

\section{METODOLOGÍA}

Para analizar las locuciones nominales del diccionario Diretes, nos preguntamos, en primer lugar, qué pruebas podrían permitirnos distinguir entre locuciones y compuestos sintagmáticos, puesto que las colocaciones nominales en el modelo de la TST no presentan problemas de categorización. Y en segundo lugar, de qué modo podríamos clasificar las locuciones nominales.

Con respecto a la primera pregunta, decidimos recurrir a la prueba de la definición ostensiva (propuesta en Barrios Rodríguez 2022). Las definiciones ostensivas se caracterizan porque asocian el referente que se pretende definir con el acto de señalar con el dedo (véase Anglada Arboix 2005: 166). De hecho, estas definiciones son las más usadas en el aprendizaje de la lengua materna, pues cuando un niño señala algo cuyo nombre desconoce, y pregunta ¿qué es eso?, los padres le responden con el nombre del referente señalado. Sirviéndonos de esta prueba, podríamos distinguir los compuestos sintagmáticos de las locuciones, y nos aproximaríamos al concepto de Buenafuentes de la Mata $(2007,2010)$ y García Page (2008), para quienes un compuesto designa algo del mundo real.

Aplicamos dicha prueba a un corpus de más de setecientas setenta y seis construcciones nominales, tomadas de Corpas Pastor (1996), Seco et alii (2004), García Page (2008), Penadés (2008) y RAE (2009). Estas, a su vez, habían sido previamente etiquetadas como posibles compuestos sintagmáticos o locuciones nominales. Tras la mencionada prueba obtuvimos seiscientas ocho palabras compuestas, y ciento sesenta y ocho locuciones nominales. Consideramos que era un conjunto suficiente para diseñar una posible clasificación de construcciones nominales para el diccionario Diretes.

11 Las FL no estándar se escriben en la lengua natural en la que se proponga su uso hasta que se demuestre que son universales, momento en el que se les adjudicará un nombre en latín, como tienen las restantes FL estándar. 
Tras haber obtenido el corpus de las locuciones nominales, y con respecto a la segunda pregunta, puesto que no hay acuerdo en la clasificación de las locuciones nominales en la bibliografía precedente, nos servimos de las pruebas de los grupos atributivos (RAE 2009: 2774) tal como también sugerimos en el mencionado trabajo (Barrios Rodríguez 2022). Entre ellas se encuentra la combinación con un verbo copulativo o semicopulativo, el que puedan funcionar como predicativo, como complemento de nombre o como predicado en construcciones no verbales. Estas pruebas permiten distinguir dos grandes grupos de locuciones nominales: las que llamamos predicativas (que son las que funcionan como adjetivos) y las designativas (que funcionan como sustantivos). Las primeras dan positivo en dos o más de las mencionadas pruebas de grupos atributivos, mientras que las designativas no logran ese mínimo.

Ejemplifiquemos el proceso que hemos seguido (tomado de Barrios Rodríguez 2022) ${ }^{12}$. Si queremos averiguar si mirlo blanco es una locución, en primer lugar comprobamos si cumple la prueba de la definición ostensiva (eso es un mirlo blanco) y vemos que: a) se cumple en una acepción (que corresponde a un tipo de mirlo), por lo que etiquetamos dicha acepción como compuesto sintagmático; b) no se cumple la prueba en una segunda acepción ('persona excepcional'), por lo que etiquetamos dicha acepción como locución.

A continuación, para saber si esta última acepción es una locución nominal predicativa o designativa, comprobamos si cumple las pruebas de atribución, como reflejamos en (5):

(5) a. *Es un poco mirlo blanco ${ }^{13}$

b. ?Se ha vuelto un mirlo blanco ${ }^{14}$

c. Ese chico es un mirlo blanco

d. ¡Ah!, ¡qué mirlo blanco!15

Siguiendo los criterios expuestos en los párrafos precedentes, como dos de las pruebas dan resultado positivo, catalogamos mirlo blanco como locución nominal predicativa.

Aplicadas estas pruebas al corpus de locuciones nominales, el resultado fue de sesenta y nueve locuciones nominales predicativas, y noventa y nueve designativas. En la Tabla 1 resumimos los tipos de prueba que hemos aplicado para cada uno de los tipos de construcción nominal:

\begin{tabular}{|l|l|l|l|}
\hline Prueba & Compuesto sintagmático & Locución predicativa & Locución designativa \\
\hline Definición ostensiva & + & - & - \\
\hline Atribución & - & + & - \\
\hline
\end{tabular}

Tabla 1: Pruebas de distinción entre compuesto y los dos tipos de locución nominal

(Barrios Rodríguez 2022)

12 Aunque Barrios (2022) finalmente se va a publicar casi simultáneamente al presente artículo, fue escrito con anterioridad.

13 Obsérvese que se rechaza la prueba del cuantificador por su significado superlativo, pues mirlo blanco se asocia a 'excepcional'.

14 En este caso consideramos que produce extrañeza la prueba del verbo de cambio porque las cualidades de las personas excepcionales se suelen ver como innatas y no como algo aprendido.

15 Tomamos el ejemplo de Crimen y Castigo de Fiódor M. Dostoievski, traducido por Rafael Cansinos Assens y publicado en Penguin Clásicos: “¡Ah, qué mirlo blanco! Peinado con raya, con sortijas... ¡Un hombre rico! ¡Oh, y qué chico tan guapito!” (https://xurl.es/fbjcq, última consulta 27/10/2021). 
Las locuciones nominales designativas, como su propio nombre indica, designan algo del mundo real. Potencialmente son expresiones referenciales y, como cualquier sustantivo con esas características, pueden ser definidas de diversos modos en un diccionario (como veremos en el apartado 6). Las predicativas, por su parte, predican algo, por lo que su definición es equivalente a un adjetivo (como veremos a continuación en el apartado 5). Respecto a unas y otras nos preguntamos cómo reflejar, de modo formal, la riqueza semántica y sintáctica de las locuciones en Diretes. Trataremos de dar respuesta a esta pregunta en los dos próximos apartados.

\section{LOCUCIONES NOMINALES PREDICATIVAS}

La pregunta que nos hicimos antes de analizar las sesenta y nueve locuciones nominales predicativas fue la siguiente: ¿Es cierto que todas ellas se relacionan con algún adjetivo? Y si es así, ¿sería útil mostrar la relación de cada locución con dichos adjetivos? Hasta ahora esta información no se encuentra en ninguno de los diccionarios de uso general precedentes, salvo, como mencionamos, mediante algunos hipervínculos, y en un único sentido, de la locución al adjetivo, pero no del adjetivo a la locución.

Consideremos el caso de canela en rama y el de mirlo blanco, locuciones usadas para hablar de personas buenas: los datos de los corpus que consultamos (CORDE, CREA, SketchEngine) confirman que la primera se dice de alguien o de algo excelente; la segunda, de alguien (pero no de algo) que es tan bueno que es raro encontrarlo. Como vemos, estas locuciones son cuasi-sinónimas, pero esta relación semántica que mantienen no se hace explícita en ningún diccionario. Por otra parte, no resulta fácil vincularlas a un adjetivo: mirlo blanco lo hemos asociado a excepcional porque consideramos que ambos contienen los rasgos 'solo uno entre muchos' y 'muy bueno'. Este último rasgo también está presente en excelente ${ }^{16}$.

Y así llegamos a la particularidad que constituye el núcleo central de nuestra propuesta de formalización y es algo que, hasta donde sabemos, no se ha indicado en la bibliografía precedente (salvo en Barrios Rodríguez 2022): existe una relación de co-hiponimia/hiperonimia entre las locuciones nominales predicativas y determinados adjetivos. En efecto, en Diretes, canela en rama y mirlo blanco se asocian a excelente y excepcional respectivamente, y estos adjetivos se asocian a bueno. Por otra parte, cada una de esas locuciones impone distintas restricciones a sus argumentos: el primero se predica tanto de personas como de situaciones (su novia es canela en rama; ese trabajo es canela en rama), mientras que el segundo se predica solo de personas (ese chico es un mirlo blanco). ¿Conviene reflejar dichas restricciones argumentales en el diccionario? Si la respuesta es afirmativa, ¿cómo hacerlo?

Con respecto a la primera cuestión, en el primer diccionario de la TST (Mel'čuk et alii 1984), ya se recoge la forma proposicional (o esquema argumental) y la definición de las palabras haciendo explícitos los rasgos semánticos de los argumentos: así, por ejemplo, en el caso de la locución à la tête ( la cabeza), se explicita que el segundo argumento corresponde a objetos o personas.

16 En la vida real lo excelente se suele dar en pocas ocasiones, por lo que lo podemos asociar a 'uno entre muchos', pero nos parece que dicha asociación no es semántica sino extralingüística, pues de hecho no hay ningún impedimento para que en un grupo numeroso de personas todas sean excelentes o para que todos los profesionales de una rama sean excelentes. 
Con respecto a la segunda cuestión, en los diccionarios de la TST se recurriría a la FL Magn ('muy') para expresar y formalizar la relación entre estas locuciones y el adjetivo bueno, tal como se ve en (6):

(6) Magn(bueno) = excelente, excepcional, extraordinario, excelso, fabuloso, formidable, genial, insuperable, maravilloso, sublime; mirlo blanco, canela en rama

La formalización de (6) es correcta pero adolece de granularidad, pues solo permite ver cierta relación de cuasi-sinonimia en ese conjunto de valores. En Diretes hemos recurrido a unas funciones léxicas no estándar (concepto presentado en el apartado 3) para las locuciones nominales predicativas (tomadas de Barrios Rodríguez 2022). Concretamente se trata de una FL no Estándar, LocPropPers, que se podría parafrasear como 'locución de propiedades de personas'; y otra FL, también no estándar, LocPropPersEntSit, que se podría parafrasear como 'locución de propiedades de personas, entidades y/o situaciones', tal como se ve en (7) y (8):

(7) LocPropPers (excepcional $)=$ mirlo blanco

(8) LocPropPersEntSit(excelente) $=$ canela en rama $^{17}$

Hay además un grupo de locuciones que predican algo de alguna(s) persona(s) en relación con otra(s) persona(s), como cabeza de turco (que paga las culpas de otros). Para ellas usamos LocPropPersPers (locución de propiedades de personas en relación con otras personas). Y hay un último grupo que predica propiedades de entidades y situaciones, pero no de personas, como juego de niños (situación fácil). Para ellas recurrimos a LocPropEnSit ${ }^{18}$. Reflejamos estas FL en (9) y (10):

(9) LocPropPersPers(culpable) = cabeza de turco; chivo expiatorio.

(10) LocPropEntSit(fácil) = juego de niños; pan comido.

Las FL de (7)-(10) reúnen estas propiedades: a) aportan información sintáctica, pues señalan que sus valores son locuciones; b) permiten llegar desde el adjetivo (excelente, excepcional, culpable, fácil) a las locuciones; c) añaden información semántica ${ }^{19}$, pues señalan propiedades de los argumentos (son personas, o situaciones, o ambas). Con respecto a este último punto, la novedad de esta formalización radica en que en lugar de reflejar las propiedades argumentales en la definición, como en à la tête (Mel'čuk et alii 1984: 53), integramos dicha información en el propio nombre de la FL.

17 La diferencia entre las dos locuciones en Diretes se hace totalmente explícita en el apartado de las definiciones que hemos redactado: mirlo blanco es "tan bueno que es raro de encontrar" y canela en rama es "de mucho valor". Obsérvese que con las locuciones ocurre algo similar a lo que ocurre con las paremias, se expresa mucho con pocas palabras, y hay un considerable margen de subjetividad. Aunque no es tarea fácil señalar su significado, el diccionario de locuciones de Seco et alii (2004) demuestra que se puede ofrecer una aproximación objetiva al sentido de las locuciones nominales. En este trabajo nos hemos basado en dicho diccionario para la redacción de nuestras definiciones.

18 Entendemos por entidad todo aquello que pertenece al mundo real que no es un ser vivo, como un objeto, una institución, una relación, etc. En nuestra propuesta no hay ningún sustantivo que designe un animal porque no los hemos encontrado en los corpus usados. Sin embargo, ha de tenerse en cuenta que en todos los casos en los que las locuciones se usan para personas podría darse el uso personificado de un nombre de animal.

19 El criterio semántico ha sido aplicado ya previamente en otros diccionarios que no son de uso general, como el de combinatoria restringida de Bosque (2004) y el de locuciones de Penadés (2008). 
Estas FL podrían parecer anómalas, con respecto a otras propuestas del modelo, pero no resultan tan particulares si se analizan los múltiples y variados casos de FL no estándar de Dicouèbe: por ejemplo, para cerveza de importación, se creó la FL Producida en el extranjero (Produite à l'étranger(bière) = d'importation, Jousse 2010: 112) ${ }^{20}$. Por otra parte, estas FL que proponemos son un tipo de hiperónimo, característica que comparte con la FL Gener, que cubre hiperónimos de expresiones nominales redundantes, como sustancia líquida, proceso de regeneración, sentimiento de vergüenza, etc. (Mel'čuk 2014: 194).

La Tabla 2 resume las FL no estándar que usamos en Diretes. En la primera columna mostramos cada FL; en la segunda, su paráfrasis; en la tercera, una locución ejemplo; en la cuarta, la definición de dicha locución; y en la quinta, el adjetivo que consideramos hiperónimo de esa locución:

\begin{tabular}{|l|l|l|l|l|}
\hline FL no Estándar & Paráfrasis & Ejemplo & Significado & Adjetivo \\
\hline LocPropPers & $\begin{array}{l}\text { Locución de propiedades de } \\
\text { personas }\end{array}$ & Mirlo blanco & $\begin{array}{l}\text { tan bueno que } \\
\text { es raro }\end{array}$ & excepcional \\
\hline LocPropPersPers & $\begin{array}{l}\text { Locución de propiedades de } \\
\text { personas en relación con otras } \\
\text { personas }\end{array}$ & $\begin{array}{l}\text { Cabeza de } \\
\text { turco, chivo } \\
\text { expiatorio }\end{array}$ & $\begin{array}{l}\text { que paga las } \\
\text { culpas de otro }\end{array}$ & culpable \\
\hline LocPropPerEntSit & $\begin{array}{l}\text { Locución de propiedades de } \\
\text { personas, entidades, situaciones }\end{array}$ & Canela en rama & muy bueno & excelente \\
\hline LocPropEntSit & $\begin{array}{l}\text { Locución de propiedades de en- } \\
\text { tidades, situaciones }\end{array}$ & $\begin{array}{l}\text { Juego de niños, } \\
\text { pan comido }\end{array}$ & muy fácil & fácil \\
\hline
\end{tabular}

Tabla 2: Propuesta de formalización de las locuciones nominales predicativas (Barrios 2022)

\section{LOCUCIONES NOMINALES DESIGNATIVAS}

Tras el análisis del apartado 5 de las locuciones que funcionan como adjetivos, nos centramos en las noventa y nueve restantes, que funcionan como sustantivos y que llamamos designativas precisamente por dicha función. La propiedad de funcionar como sustantivo la comparten con los compuestos sintagmáticos, lo que genera buena parte de la confusión mencionada en la bibliografía (una buena síntesis de estos problemas se puede ver en Orduña López 2019). Como hemos adelantado, aquí nos hemos servido de la regla de la definición ostensiva (\#eso es sapos y culebras) porque no aceptan un uso referencial, uso típico de los compuestos sintagmáticos ${ }^{21}$.

La mayoría de estas locuciones son difícilmente asociables a un único sustantivo, por lo que las asociamos a una paráfrasis, y en muchos casos analizamos la polisemia: así, ojo del huracán, es una palabra compuesta cuando designa la zona de relativa calma del centro del huracán (acepta la definición ostensiva), y se comporta como una locución nominal designativa cuando designa el centro de una situación polémica o conflictiva $(D L E)^{22}$.

20 En su trabajo Jousse recopila otros muchos ejemplos.

21 Obsérvese que, aunque se puede decir "eso es un jarro de agua fría", o "eso son sapos y culebras", estos enunciados no constituyen una definición ostensiva, simplemente sirven para decir que esa crítica concreta tiene todos los rasgos de lo que entendemos por jarro de agua fría; o que esas palabras presentan todas las características típicas de lo que se conoce por sapos y culebras. La definición ostensiva precisa de un referente del mundo real que se pueda señalar, de ahí que hablemos de uso referencial.

22 Última consulta 27/10/2021. 
Presentamos algunas de las locuciones designativas de nuestro corpus con las que hemos trabajado en la Tabla 3:

\begin{tabular}{|l|l|l|}
\hline Locución nominal & Definición & Ejemplo de uso nominal \\
\hline bautismo de fuego & prueba de iniciación & $\begin{array}{l}\text { El paracaidista vivió su bautismo de } \\
\text { fuego }\end{array}$ \\
\hline boca del lobo & lugar/situación difícil & No vaya a meterse en la boca del lobo \\
\hline buena mano & habilidad manual o psicológica & Tiene buena mano para la cocina \\
\hline cascarón de nuez & embarcación frágil & Viajaban en un cascarón de nuez \\
\hline cuesta de enero & escasez económica & La cuesta de enero se presenta empinada \\
\hline don de gentes & $\begin{array}{l}\text { cualidad para conquistar a los } \\
\text { demás }\end{array}$ & Tiene don de gentes \\
\hline el más allá & vida después de la muerte & Creer en el más allá \\
\hline el qué dirán & opinión de los demás & Todo lo hace por el qué dirán \\
\hline el otro mundo & cielo, vida eterna & Se nos ha ido al otro mundo \\
\hline fuerza mayor & obligación no deseada & Decidido por fuerza mayor \\
\hline $\begin{array}{l}\text { gallina de los huevos } \\
\text { de oro }\end{array}$ & algo que da dinero fácil & Mató la gallina de los huevos de oro \\
\hline gramática parda & habilidad natural & Sabe gramática parda \\
\hline horas muertas & tiempo perdido & Eliminar las horas muertas \\
\hline la cuenta de la vieja & cuentas muy básicas & Hace siempre la cuenta de la vieja \\
\hline lengua de trapo & $\begin{array}{l}\text { habla de los niños muy peque- } \\
\text { ños }\end{array}$ & Tiene lengua de trapo \\
\hline lo divino y lo humano & muchos temas variados & Hablamos de lo divino y lo humano \\
\hline malas artes/juego sucio & acto inmoral & Lo consiguió con malas artes/juego sucio \\
\hline patata caliente & problema incómodo & Te pasan la patata caliente \\
\hline buenas palabras & halago falso & $\begin{array}{l}\text { No me vengas ahora con buenas } \\
\text { palabras }\end{array}$ \\
\hline
\end{tabular}

Tabla 3: Locuciones nominales designativas (datos tomados de Barrios 2022)

Como se puede apreciar, la Tabla 3 tiene solo tres columnas, y la segunda recoge el grupo nominal que parafrasea su sentido. Falta la columna del sustantivo (equivaldría en la Tabla 2 a la columna del adjetivo) porque, como hemos adelantado, para este tipo de locuciones resulta difícil encontrar solo uno que pueda funcionar como hiperónimo. En los casos en los que por ahora hemos podido asociarlo a un sustantivo (como en habilidad vs. buena mano), añadimos la FL LOCUCIÓN, creada ad hoc para las locuciones designativas. Esta FL permite la misma funcionalidad que la mostrada en (7)-(10), aunque no aporta granularidad semántica ni sintáctica, tal como se ve en (11):

(11) LOCUCIÓN(habilidad) = buena mano

\section{RESULTADOS}

Tras resumir los criterios usados para la clasificación de las construcciones nominales de nuestro corpus, y muy particularmente de las locuciones nominales, presentamos cómo se 
reflejan en el diccionario Diretes. En él se conserva la información de los diccionarios tradicionales, pero además, como en todos los diccionarios de la TST, se añade una entrada de diccionario para cada locución, en la que reflejamos su definición y las FL correspondientes.

Así, por ejemplo, si en el buscador se teclea la palabra hombre se ofrece una lista con todas las entradas del diccionario en las que se encuentra esta palabra. En este caso concreto, se muestra la palabra hombre, diversas locuciones, como hombre anuncio, hombre bala, hombre de negocios, hombre de paja, etc., la fórmula ;hombre al agua! y el acto de habla estereotipado ipobre hombre!

Los resultados se ofrecen por orden categorial (los sustantivos agrupados, las locuciones de cada tipo, etc.) y, dentro de cada categoría, por orden alfabético. Cada una de esas expresiones cuenta con un hipervínculo que permite el acceso a la entrada correspondiente, como se ve en la Ilustración 1:

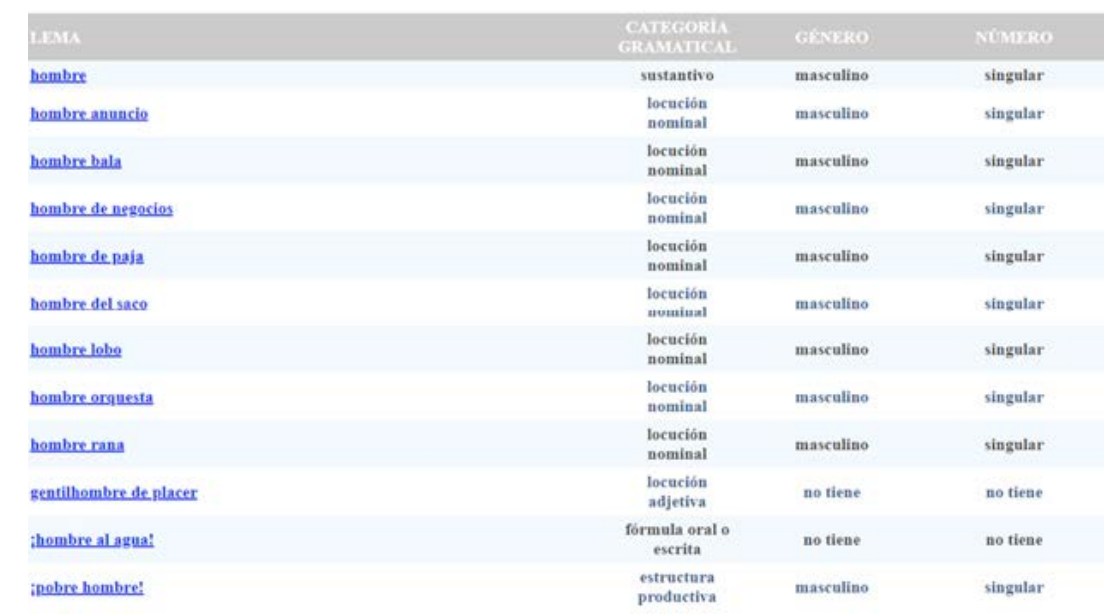

Ilustración 1: Resultados de la búsqueda hombre en Diretes (27/10/2021)

Si se accede a la entrada hombre de paja, se aprecia que contiene una definición mínima, una expandida (que es una definición con rasgos adicionales) y una etiqueta semántica (o categoría semántica) (acerca de estos conceptos, véase Barrios, 2019b). La locución remite al hiperónimo responsable, como se ve en la Ilustración 2:

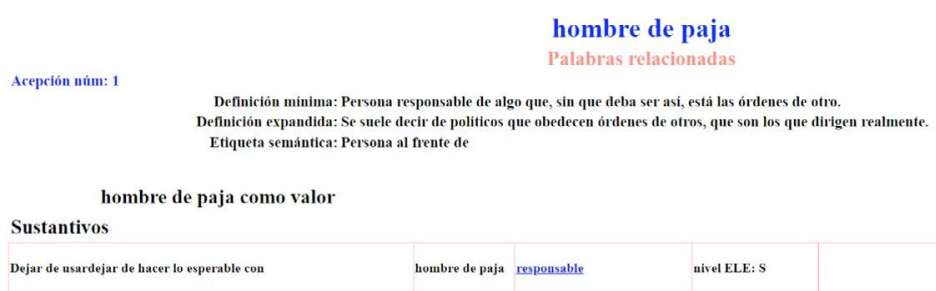

Ilustración 2: Entrada hombre de paja en Diretes (27/10/2021) 
Lo novedoso es que la formalización de Diretes permite que se recojan los sentidos figurados asociados a responsable, de tal manera que también se llega a hombre de paja desde la entrada responsable (no como en la mayoría de los diccionarios de uso, en los que se accede desde hombre o desde paja).

Como el diccionario Diretes está en curso, algunos resultados se acompañan de la etiqueta "Sin asignar" (como ocurre en la definición mínima de dicha ilustración). Con eso se indica que esa entrada aún no se ha desarrollado. El mismo sentido tiene la letra $\mathrm{S}$ en las casillas del nivel de ELE (Español como Lengua Extranjera) (véase cuarta columna). Como se puede apreciar, aunque la entrada responsable aún no se haya redactado, se ha recuperado de modo automático la información que se ha introducido en la entrada hombre de paja, como muestra la Ilustración 3:

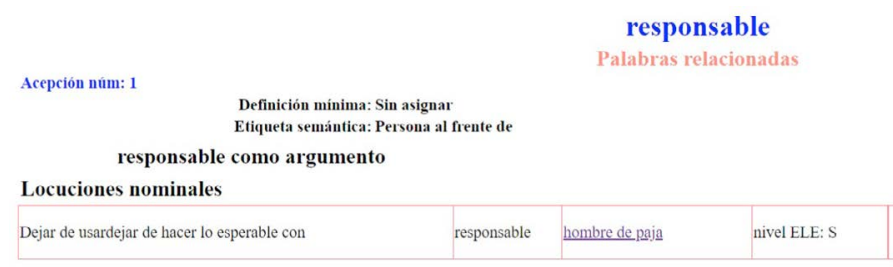

Ilustración 3: Vínculo a hombre de paja desde la entrada responsable del diccionario Diretes $(27 / 10 / 2021)$

Nos planteábamos en el apartado 2 cómo relacionar mirlo blanco con canela en rama. ¿Una persona que no recuerde estas locuciones pero que sepa que se asocian a muy bueno, puede encontrarlas en Diretes? La respuesta es afirmativa, pues en este diccionario, al teclear bueno se encuentra excelente, excepcional ${ }^{23}$, extraordinario, etc., como refleja la Ilustración 4:

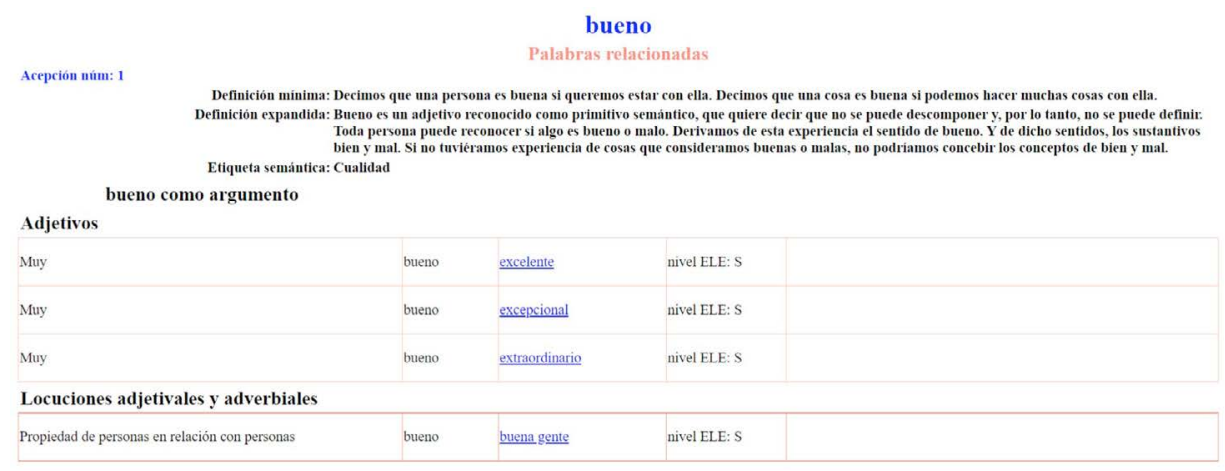

Ilustración 4: Parte de la entrada bueno del diccionario Diretes (27/10/2021)

23 En Diretes se han recogido dos acepciones de excepcional, la primera asociada a raro, y la segunda a excelente: aunque esta última aún no la recoja el $D L E$ (última consulta el 27/10/2021), la hemos considerado frecuente en la conciencia de los hablantes. 
Si se hace clic en excelente (primera fila de la Ilustración 4) se despliega la información de la Ilustración 5, en la que se puede ver que se podrá llegar tanto a canela en rama como a muy bueno:

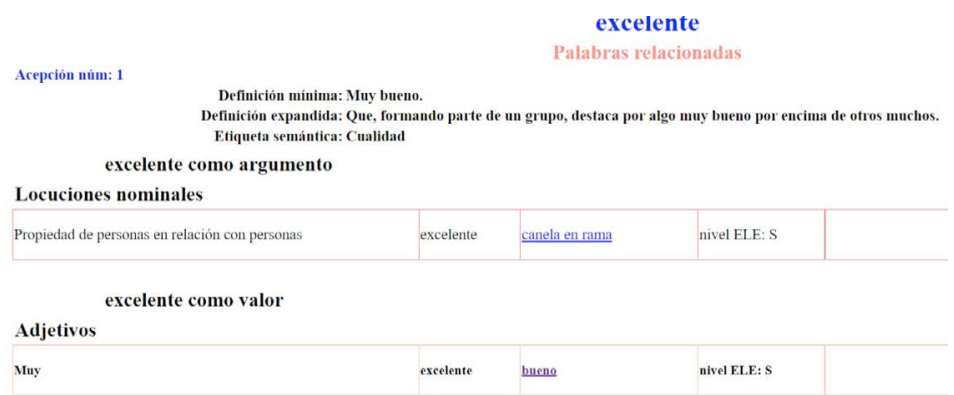

Ilustración 5: Entrada de excepcional en Diretes (27/10/2021)

Pero, ¿qué ocurre con la expresión canela en rama cuando designa un tipo de canela? $\mathrm{Si}$ en el buscador tecleamos canela se ofrecen los vínculos a las entradas de las palabras compuestas canela en rama, canela en polvo y canela molida, y a la locución nominal predicativa canela en rama, como se ve en la Ilustración 6:

\begin{tabular}{|c|c|c|c|}
\hline LDMA & $\begin{array}{l}\text { CATHEORIA } \\
\text { GRAMATICAL }\end{array}$ & GíNDRO & NÚMIDRO \\
\hline canela & sustantivo & femenino & singular \\
\hline canela en polvo & $\begin{array}{c}\text { palabra } \\
\text { compuesta }\end{array}$ & femenino & singular \\
\hline canela en rama & $\begin{array}{c}\text { palabra } \\
\text { compuesta }\end{array}$ & femenino & singular \\
\hline canela molida & $\begin{array}{c}\text { palabra } \\
\text { compuesta }\end{array}$ & femenino & singular \\
\hline canela en rama & $\begin{array}{c}\text { locución } \\
\text { nominal } \\
\text { predicativa }\end{array}$ & $\begin{array}{l}\text { concuerda con los dos } \\
\text { géneros }\end{array}$ & $\begin{array}{c}\text { concuerda con los dos } \\
\text { números }\end{array}$ \\
\hline
\end{tabular}

Ilustración 6: Resultados de canela en Diretes

La expresión canela en rama la hemos categorizado como palabra compuesta (tercera fila de la Ilustración 6) si designa una especia, y locución nominal predicativa (quinta fila de la Ilustración 6) si se predica de alguien o algo excepcionalmente bueno. Los compuestos sintagmáticos canela en rama, canela en polvo y canela molida están formalizados como un tipo de canela; y los compuestos canela en polvo y canela molida están asociados entre sí por la FL Syn (sinónimo), como se puede ver, respectivamente, en las primeras y en la última fila de la Ilustración 7: 


\begin{tabular}{|c|c|c|c|}
\hline _TIPO DE & canela (s. f. sg.) & 1 - Especia & canela en rama (p. c. f. sg.) 1 - Especia \\
\hline _TIPO DE & canela (s. f. sg.) & 1 - Especia & canela molida (p. c. f. sg.) 1 - Especia \\
\hline Real1 & canela ( s. f. sg.) & 1 - Especia & agregar (v. - -) 1 - Acción \\
\hline Real1 & canela (s. f. sg.) & 1 - Especia & añadir ( v. - -) 1 - Acción \\
\hline Real1 & canela (s.f. sg.) & 1 - Especia & condimentar ( v. . - ) 1-Sin asignar \\
\hline Real1 & canela (s. f. sg.) & 1 - Especia & consumir ( v. ..) 1-Sin asignar \\
\hline Real1 & canela (s. f. sg.) & 1 - Especia & echar ( v. - -) 1 - Sin asignar \\
\hline Real1 & canela (s. f. sg.) & 1 - Especia & espolvorear (v. - ) 1 - Sin asignar \\
\hline Real1 & canela ( s. f. sg.) & 1 - Especia & incorporar (v. - ) 1 - Sin asignar \\
\hline Real1 & canela (s. f. sg.) & 1-Especia & poner $(v, \ldots) 1-$ Sin asignar \\
\hline Real1 & canela ( s. f. sg.) & 1 - Especia & sazonar ( v. - - ) 1 - Sin asignar \\
\hline Real2 & canela ( s. f. sg.) & 1 - Especia & llevar (v. - - ) 1 - Sin asignar \\
\hline Syn & $\begin{array}{l}\text { canela en polvo ( } \\
\text { Especia }\end{array}$ & (p. c. f. sg.) 1 - & canela molida (p. c. f. sg.) 1 - Especia \\
\hline Tipo de-forma & canela (s. f. sg.) & 1 - Especia & en polvo (loc. adj...) 1 - Sin asignar \\
\hline Tipo de-forma & canela (s. f. sg.) & 1 - Especia & en rama (loc. adj. .-) 1 - Sin asignar \\
\hline
\end{tabular}

Ilustración 7: Algunas relaciones asociadas al sustantivo canela y a la palabra compuesta canela en polvo

Por otra parte, las locuciones canela en rama y mirlo blanco comparten la FL LocPropPersPers, para los adjetivos excelente y excepcional, ambos cuasi-sinónimos, como se puede ver en la Ilustración 8:

\begin{tabular}{|c|c|c|}
\hline _LocPropPersPers & excelente (adj. c. c.) 1 - Cualidad & $\begin{array}{l}\text { canela en rama (loc. n. pred. c. c.) } 1 \text { - } \\
\text { Cualidad }\end{array}$ \\
\hline Synn & excelente (adj. c. c.) 1 - Cualidad & excepcional (adj. c. c.) 2 - Cualidad \\
\hline Synn & excelente (adj.c. c.) 1 - Cualidad & extraordinario (adj. c. c.) 1 - Cualidad \\
\hline _LocPropPersPers & excepcional (adj. c. c.) 2 - Cualidad & $\begin{array}{l}\text { mirlo blanco (loc. n. pred. m. sg.) } 1 \text { - } \\
\text { Cualidad }\end{array}$ \\
\hline
\end{tabular}

Ilustración 8: Algunas relaciones léxico-semánticas asociadas excelente y excepcional

Estos ejemplos permiten ver una de las ventajas de lo que proponemos aquí: la vinculación de palabras y locuciones que comparten rasgos semánticos, y su formalización con FL no estándar, permiten el acceso desde el sentido y desde la forma. La otra ventaja de nuestra propuesta es que algunas entradas ganan en claridad, pues permiten reflejar la diversa tipología de las locuciones, algo especialmente importante en fraseología.

Para terminar de ilustrar las ventajas de la categorización gramatical y de las formalizaciones que proponemos, consideremos el caso de falso. En Diretes se puede llegar a las locuciones cuyo significado contenga el rasgo 'falso', como ocurre con las locuciones nominales predicativas caballo de Troya, castillo de naipes, cuento chino, flor de un día, música celestial, un quiero y no puedo, o figura decorativa. Esto, hasta donde sabemos, solo lo ofrece el DUE. La Ilustración 9 lo muestra: 


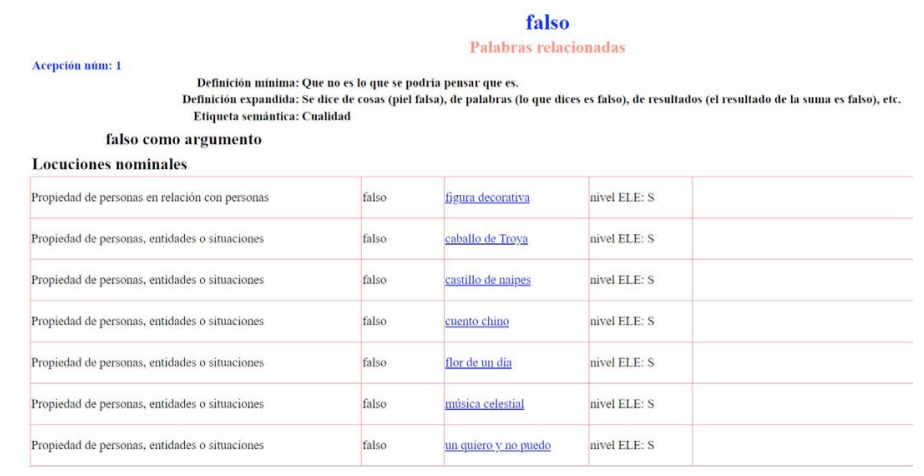

Ilustración 9: Locuciones nominales predicativas asociadas a falso en Diretes

Pero, además, hay una Función Léxica que asocia el adjetivo falso al sustantivo falsedad: la $\mathrm{FL} \mathrm{S}_{0}$ (que significa 'sustantivo derivado'). Por eso en la búsqueda falso como valor, se ofrece el vínculo a falsedad. Si vamos a la entrada de falsedad apreciamos que el buscador permite llegar a las locuciones nominales designativas becerro de oro, buenas palabras y malas lenguas, como se muestra en la Ilustración 10:

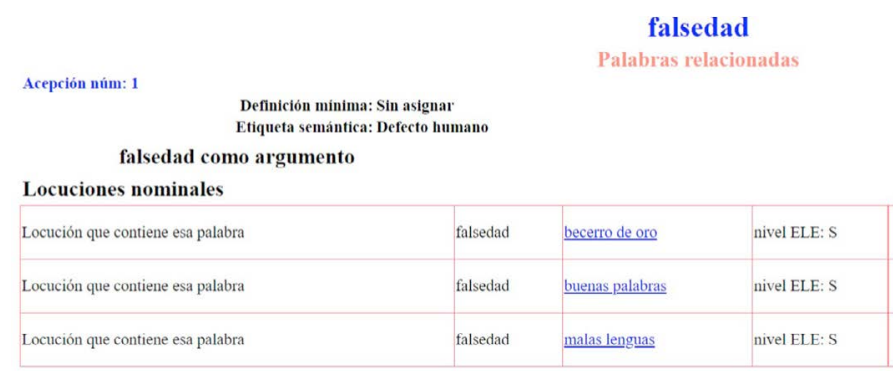

Ilustración 10: Locuciones nominales designativas asociadas a falsedad en Diretes

Dicho con otras palabras, la distinción entre locuciones nominales designativas y predicativas tiene como consecuencia que en el diccionario se seleccione su hiperónimo como sustantivo o adjetivo respectivamente.

\section{CONCLUSIONES}

Concluimos pues retomando las preguntas que planteábamos al comienzo de este trabajo: la implementación en Diretes de la tipología de locuciones nominales que distingue entre predicativas (como las de la Ilustración 9) y designativas (como las de la Ilustración 10) ha sido validada en un corpus de más de seiscientas construcciones nominales. Por otra parte, en el diccionario ha resultado muy útil la formalización de las locuciones nominales con las funciones léxicas no estándar que hemos creado ad hoc.

Hemos verificado que las locuciones nominales predicativas son hipónimos de adjetivos: obsérvese en la Ilustración 9 que se predican del adjetivo falso y que se podrían parafra- 
sear frecuentemente como tal ("eso es un cuento chino"/ "eso es falso"). Las locuciones nominales designativas, sin embargo, son hipónimos de sustantivos o de grupos nominales: obsérvese que en la Ilustración 10 se asocian al sustantivo falsedad y se podría sustituir por él ("y ahora viene con esas buenas palabras"/ "y ahora viene con esa falsedad").

El hecho de que hayamos podido implementar esta propuesta en los esquemas rígidos y necesariamente sin ambigüedades de la planta de un diccionario electrónico ha sido suficiente, por el momento, como para que decidamos seguir implementándola. Desde un punto de vista práctico, la ventaja de la formalización que proponemos es que permite la navegación en un diccionario electrónico no solo en sentido semasiológico (en la entrada del diccionario mirlo está la locución mirlo blanco) sino también en un sentido onomasiológico: desde la entrada bueno se puede llegar a la entrada excelente y excepcional; desde excelente se puede llegar a canela en rama; y desde excepcional se puede llegar a mirlo blanco; desde las entradas de cualquiera de estas dos locuciones se puede llegar a la otra, y también a excelente y excepcional, y desde estas últimas a bueno.

Nuestro proyecto aún está en curso y debemos formalizar aún más de mil locuciones nominales, por lo que es esperable que haya modificaciones, o que encontremos nuevos patrones semánticos. Con todo, pensamos que hemos dado un primer paso hacia una tipología formal de las locuciones nominales.

\section{REFERENCIAS BIBLIOGRÁFICAS}

Alonso Ramos, M. (2009). "Delimitando la intersección entre composición y fraseología”, LEA: Lingüistica Española Actual, 31(2), pp. 243-275.

Anglada Arboix, E. (2005). Lexicografía española. Barcelona: Universitat de Barcelona.

Apresjan, J., I. Boguslavsky, L. L. Iomdin, A. Lazursky, V. Sannikov, V. Sizov y L. Tsinman (2003). "ETAP-3 Linguistic Processor: a Full-Fledged NLP Implementation of the Meaning-Text Theory". Moscú: Institute for Information Transmissions Problems: http://proling.iitp.ru/bibitems/ETAP and_MTT.pdf (26/10/2021).

Barrios Rodríguez, M.A. (2010). El dominio de las funciones léxicas en el marco de la Teoría SentidoTexto. Estudios de Lingüística del español (ELiEs), vol 30. http://elies.rediris.es/elies30/index30. html (26/10/2021).

Barrios Rodríguez, M.A. (2015). Las colocaciones del español. Madrid: Arco/Libros.

Barrios Rodríguez, M.A. (2019a). “¿Aún queda alguien para quien no exista un diccionario? Diretes, un diccionario electrónico apto para máquinas". En Cazorla, M.C., M.A. García Aranda, \& P. Nuño (eds.). Lo que hablan las palabras. Estudios de lexicología, lexicografía y gramática en honor de Manuel Alvar Ezquerra. Lugo: Axac, pp. 33-46.

Barrios Rodríguez, M.A. (2019b). "Minimal and inverse definitions: a semi-experimental proposal when building a Spanish dictionary with semantic primes and molecules". En Peeters, B., K. Mullan y L. Sadow (eds.). Studies in Ethnopragmatics, (Cultural) Semantics, and Minimal English. Berlin: Springer, pp. 191-212.

Barrios Rodríguez, M. A. (2020). "The making of the Diretes dictionary". En Gavriilidou, Z., M. Mitsiaki y A. Fliatouras (eds.). Lexicography for inclusion. EURALEX Proceedings. XIX Congress of the European Association for Lexicography. Alexandroupolis: Democritus University of Thrace/ European Association for Lexicography, pp.13-22.

Barrios Rodríguez, M. A. (2022). Las colocaciones nominales: una aproximación desde la Teoría Sentido-Texto. Santiago de Compostela: Verba. Anuario Galego de Filoloxía, Anexo 82.

Barrios Rodríguez, M. A. e I. Boguslavsky. (2019). "A Spanish E-dictionary: Standard and Nonstandard Lexical Functions". En Kahane S. y K. Gerdes (eds.). Depling 2019 Proceedings. 
Stroudsburg: Association for Computational Linguistics (ACL): https://www.aclweb.org/ anthology/W19-7700/ (26/10/2021).

Bosque-Gil, J., J. Gracia, E. Montiel-Ponsoda y G. Aguado-de-Cea (2016). "Modelling multilingual lexicographic resources for the web of data: the $\mathrm{K}$ dictionaries case". En Kenerman, I., I. Kosem, S. Krek y L. Trap-Jensen (eds.). Proceedings GLOBALEX Lexicographic Resources for Human Language Technology: http://www.lrec-conf.org/proceedings/lrec2016/workshops/ LREC2016Workshop-GLOBALEX_Proceedings-v2.pdf (26/10/2021).

Bosque-Gil, J., J. Gracia, E. Montiel-Ponsoda y A. Gómez-Pérez (2018). "Models to represent linguistic linked data", Natural Language Engineering, 24(6), pp. 811-859. https://zaguan.unizar. es/record/84323/files/texto_completo.pdf (26/10/2021).

Bosque-Gil, J., D. Donke, J. Gracia y I. Kernerman (2019). "Validating the Ontolex-lemon with K-dictionaries Multilingual Data". En Kosem, I. et alii. Electronic lexicography in the 21 st century. Proceedings of e-Lex 2019 Conference. Brno: Lexical Computing, pp. 726-746: https:// elex.link/elex2019/wp-content/uploads/2019/09/eLex_2019_41.pdf (26/10/2021).

Bosque Muñoz, I. (1989). Las categorías gramaticales. Relaciones y diferencias. Madrid: Síntesis.

Bosque Muñoz, I. (1999). "El nombre común”. En Bosque Muñoz, I. y V. Demonte Barreto (eds.). Gramática descriptiva de la lengua española. Madrid: Espasa, pp. 3-75.

Bosque Muñoz, I. (2004). Redes. Diccionario combinatorio del español contemporáneo. Madrid: S.M.

Bosque, I. (2017). "Posibilidades de un diccionario onomasiológico de modismos para estudiantes de ELE. En Ainciburu, M. C. y C. Fernández Silva (eds.). La adquisición de la lengua española: aprendizaje, enseñanza, evaluación. Estudios en Homenaje a Marta Baralo Ottonello. Buenos Aires: Autores de Argentina, pp. 26-37.

Buenafuentes de la Mata, C. (2007). Procesos de gramaticalización y lexicalización en la formación de compuestos en español. Barcelona: Universidad Autónoma de Barcelona.

Buenafuentes de la Mata, C. (2010). La composición sintagmática en español. Ciluenga: San Millán de la Cogolla.

Buendía-Castro, M. (2020) Verb collocations in dictionaries and corpus: an integrated approach for translation purposes. Berlín: Peter Lang.

Bustos Gisbert, E. (1986). La composición nominal en español. Salamanca: Ed. Universidad de Salamanca.

Cabezas García, M. (2020). Los términos compuestos desde la Terminología y la Traducción. Berlin: Peter Lang.

Casares, J. (1992) [1969]. Introducción a la lexicografía moderna. Madrid: Consejo Superior de Investigaciones Científicas.

Corpas Pastor, G. (1996). Manual de fraseología española. Madrid: Gredos.

Fonseca, A., F. Sadat y F. Laureau (2016a). "A Lexical Ontology to Represent Lexical Functions". En LREC (ed.). Proceedings of the 2nd Workshop on Language and Ontologies (OntoLex): http:// www.lrec-conf.org/proceedings/lrec2016/workshops.html (26/10/2021).

Fonseca, A., F. Sadat y F. Laureau (2016b). "Lexform: A lexical function ontology model”. En COLING 2016 Organizing Committee (ed.). Proceedings of the Workshop on Cognitive Aspects of the Lexicon. Osaka: Aclanthology, pp. 145-155: https://aclanthology.org/W16-5320.pdf (26/10/2021).

Fonseca, A., F. Sadat y F. Laureau (2017). "Combining Dependency Parsing and a Lexical Network Based on Lexical Functions for the Identification of Collocations". En Mitkov, R. (ed.). Computational and Corpus-Based Phraseology. EUROPHRAS 2017. Lecture Notes in Computer Science, Vol. 10596. Cham: Springer. https://link.springer.com/chapter/10.1007\% 2F978-3-319-69805-2_31 (26/10/2021).

García Page, M. (2008). Introducción a la fraseología española. Estudio de las locuciones. Barcelona: Antrophos.

García Page, M. (2011). “Hombre rana, hombre clave, ¿un mismo fenómeno?”, Verba, 38, pp. 127170. 
García Page, M. (2012). "Pourquoi perrito caliente nest pas la même chose que patata caliente ? Du composè á la locution nominale”. En Blasco, X., S. Fuentesy S. Mejri (eds.). Les locutions nominales en langue générale. Barcelona: Universitat Autònoma, pp. 79-108.

Ionov, M., J. P. McCrae, C. Chiarcos, T. Declerck, J. Bosque-Gil y J. Gracia (2020). Proceedings of the 7th Workshop on Linked Data in Linguistics (LDL-2020): https://www.aclweb.org/anthology/2020. ldl-1.0.pdf (26/10/2021).

Koike, K. (2001). Colocaciones léxicas en el español actual: estudio formal y léxico-semántico. Madrid: Universidad de Alcalá/ Takushoku University.

Koike, K. (2009). "Las locuciones nominales del español”. Takushoku Language Studies, 12(1), pp. $1-45$.

Kouvara, E., M. González, J. Grosse and R. Sauri (2020). "Determining Differences of Granularity between Cross-Dictionary Linked Senses" En Gavriilidou, Z., M. Mitsiaki, A. Fliatouras (eds.). Lexicography for inclusion. EURALEX Proceedings. XIX Congress of the European Association for Lexicography. Alexandroupolis: Democritus University of Thrace/ European Association for Lexicography, pp.109-118.

Liu, L. (2018). Colocaciones nominales en español y en chino: estudio contrastivo (tesis inédita). Madrid: Universidad Complutense de Madrid.

McCrae, J. P., J. Bosque-Gil, J. Gracia, P. Buitelaar, P. Cimiano (2017). "The Ontolex-Lemon model: development and applications". Proceedings of eLex 2017 Conference: http://john.mccr.ae/papers/ mccrae2017ontolex.pdf (26/10/2021).

Melčuk, I. (1984). Dictionnaire explicatif et combinatoire du français contemporain. Recherche Lexico-Sémantiques I. Montreal : Les Presses de l'université de Montréal.

Melčuk, I. (1996). "Lexical functions: A tool for the description of lexical relations in a lexicon". En L Wanner (ed.). Lexical Functions in Lexicography and Natural Language Processing. Amsterdam/ Philadelphia: John Benjamin, pp. 37-102.

Mel’čuk, I. (2006): «Parties du discours et locutions», Bulletin de la Société de Linguistique de Paris, 101(1), pp. 29-65.

Mel'čuk I. (2014). Semantics. From meaning to text. Volume 3. John Benjamins Publishing Company. Amsterdam/Philadelphia.

Mel'čuk I., and L. Wanner (1996). "Lexical Functions and Lexical Inheritance". En L. Wanner (ed.). Lexical Functions in Lexicography and Natural Language Processing. Amsterdam: John Benjamins Publishing Company, pp. 209-278.

Mel'čuk I., and L. Wanner (2001). "Towards a Lexicographic Approach to Lexical Transfer in Machine Translation (Illustrated by the German-Russian Language Pair)". Machine Translation, 16, pp. 21-87.

Mihindukulasooriya, N., G. Rossiello, P. Kapanipathi, I. Abdelaziz, S. Ravishankar, M. Yu, A. Gliozzo, S. Roukos, A. Gray. (2020). "Leveraging Semantic Parsing for Relation Linking over knowledge bases": https://www.researchgate.net/publication/344276832_Leveraging_Semantic_Parsing_for_ Relation_Linking_over_Knowledge_Bases (26/10/2021).

Mikolov, T., K. Chen, G. Corrado y J. Dean (2013). "Efficient Estimation of Word Representations in Vector Space", Computation and Language: https://arxiv.org/abs/1301.3781 (26/10/2021).

Moliner, M. (2016). Diccionario de uso del español. Madrid: Gredos.

Orduña López, J. L. (2019). ¿Locuciones nominales o compuestos sintagmáticos? A propósito del español del valle de Aburrá. Íkala. Revista de lenguaje y cultura, XXVI (3), pp. 619-636.

Penadés Martínez, I. (2008). Diccionario de locuciones nominales, adjetivas y pronominales para la enseñanza del español. Madrid: Arco/Libros.

Pilehvar, M. T., Camacho-Collados, J. (2019). "WiC: The word-in-context dataset for evaluating context-sensitive meaning representations". Proceedings of the NAACL-HLT Conference: https:// aclanthology.org/N19-1128.pdf (26/10/2021). 
Polguère, A. (2007). "Lexical function standardness". En Leo Wanner (ed.). Selected Lexical and Grammatical Issues in the Meaning-Text Theory. In Honour of Igor Melčuk. Amsterdam/ Philadelphia: John Benjamin, pp. 43-95.

Polguère, A. (2015). "Non-compositionalité: ces sont toujours les locutions faibles qui trinquent". Verbum XXXVII(2). 257-280.

Real Academia Española. (2009). Nueva gramática de la lengua española. Madrid: Espasa.

Roukos, S. (2014). "IBM Cognitive Computing. An NLP Renaissance!". Proceedings of the Conference on Empirical Methods in Natural Language Processing (EMNLP). Doha: Association for Computational Linguistics, p. 1: https://emnlp2014.org/papers/pdf/EMNLP2014001.pdf $(26 / 10 / 2021)$.

Seco, M. , O. Andrés y G. Ramos (2004). Diccionario fraseológico documentado del español actual. Locuciones y modismos españoles. Madrid: Aguilar.

Torner, S. y E. Bernal (eds.) (2017). Collocations and other lexical combinations in Spanish. New York: Routledge.

Wanner, L. y F. Laureau. (2009). "Applying the Meaning-Text Theory Model to Text Synthesis with Low- and Middle-Density Languages in Mind". En Nirenburg, S. (ed). Language Engineering for Lesser-Studied Languages. Amsterdam/Berlín/Oxford/okyo/Washington D. D.: IOS Presses, pp. 207-242.

Zuluaga, A. (1980). Introducción al estudio de las expresiones fijas. Verlag Peter D. Lang: Frankfurt. 